\title{
Breast Cancer Histopathological Image Classification: A Deep Learning Approach
}

\author{
Mehdi Habibzadeh Motlagh ${ }^{1}$, Mahboobeh Jannesari ${ }^{2}$, HamidReza Aboulkheyr ${ }^{1}$, Pegah Khosravi ${ }^{3}$, Olivier \\ Elemento $^{3, *}$, Mehdi Totonchi ${ }^{1,2, *}$, and Iman Hajirasouliha ${ }^{3, *}$ \\ ${ }^{1}$ Department of Stem Cells and Developmental Biology, Cell Science Research Center, Royan Institute for Stem \\ Cell Biology and Technology, ACECR \\ ${ }^{2}$ Department of Genetics, Reproductive Biomedicine Research Center, Royan Institute for Reproductive \\ Biomedicine, ACECR, Tehran, Iran \\ ${ }^{3}$ Englander Institute for Precision Medicine, The Meyer Cancer Center, Institute for Computational Biomedicine, \\ Department of Physiology and Biophysics, Weill Cornell Medicine of Cornell University, NY, USA. \\ *co-corresponding authors
}

\begin{abstract}
Breast cancer remains the most common type of cancer and the leading cause of cancer-induced mortality among women with 2.4 million new cases diagnosed and 523,000 deaths per year. Historically, a diagnosis has been initially performed using clinical screening followed by histopathological analysis. Automated classification of cancers using histopathological images is a chciteallenging task of accurate detection of tumor sub-types. This process could be facilitated by machine learning approaches, which may be more reliable and economical compared to conventional methods.

To prove this principle, we applied fine-tuned pre-trained deep neural networks. To test the approach we first classify different cancer types using 6,402 tissue micro-arrays (TMAs) training samples. Our framework accurately detected on average $99.8 \%$ of the four cancer types including breast, bladder, lung and lymphoma using the ResNet V1 50 pre-trained model. Then, for classification of breast cancer sub-types this approach was applied to 7,909 images from the BreakHis database. In the next step, ResNet V1 152 classified benign and malignant breast cancers with an accuracy of $98.7 \%$. In addition, ResNet V1 50 and ResNet V1 152 categorized either benign- (adenosis, fibroadenoma, phyllodes tumor, and tubular adenoma) or malignant(ductal carcinoma, lobular carcinoma, mucinous carcinoma, and papillary carcinoma) sub-types with $94.8 \%$ and $96.4 \%$ accuracy, respectively. The confusion matrices revealed high sensitivity values of $1,0.995$ and 0.993 for cancer types, as well as malignantand benign sub-types respectively. The areas under the curve (AUC) scores were $0.996,0.973$ and 0.996 for cancer types, malignant and benign sub-types, respectively. Overall, our results show negligible false negative (on average 3.7 samples) and false positive (on average 2 samples) results among different models. Availability: Source codes, guidelines and data sets are temporarily available on google drive upon request before moving to a permanent GitHub repository.
\end{abstract}

\section{INTRODUCTION}

Recent global cancer statistics reported that breast cancer is still the most common cancer type and the leading cause of cancer-induced mortality among women, worldwide, with 2.4 million new cases and 523,000 deaths per year [19].

Histopathological classification of breast carcinoma is typically based on the diversity of the morphological features of the tumors, comprising 20 major tumor types and 18 minor sub-types ([37]). Approximately $70-80$ percent of all breast cancers belongs to either one of the two major histopathological classes, namely invasive ductal carcinoma (IDC) or invasive lobular carcinoma (ILC) ([39], [57]). The IDC class is divided into five different carcinoma sub-types including tubular, medullary, papillary, mucinous and cribriform carcinomas, while benign types of breast cancer contains adenosis, fibroadenoma, phyllodes tumor and tubular adenoma. More importantly, identification of minor tumor sub-types known as special tumor types provides clinically useful information to determine an effective therapy. For instance, accurate diagnosis of tubular and cribriform breast carcinoma can lead to employment of an appropriate treatment and increased overall survival rate. ([57], [12]). A wide range of clinical studies reported lack of complete overlap between immunohistochemically and molecular classification of breast cancer $([\overline{6}])$. However, in 2011 St Gallen International Expert Consensus validated application of immunohistochemistry for identification breast cancer sub-types ([20]). Because of the extensive heterogenecity in breast cancer, and limited predictive power of the histopathological classification, a comprehensive approach for accurate evaluation of cell morphological features is highly required.

To maximize knowledge of cancer detection and interpretation, pathologists have to study large numbers of tumor tissue slides. Additionally, quantification of different parameters (e.g. mitotic counts, surface area and cell size) and evaluation of immunohistochemical molecular markers can be complicated and time consuming. Manual inspection methods introduce three inevitable types of error including statistical, distributional and human errors in low magnification images. These problems adversely affect the accuracy of the differential classification in conventional cancer diagnosis. Therefore, an automated and reproducible methodology could tackle the aforementioned obstacles more effectively.

Computer-aided diagnosis (CAD) established methods for robust assessment of medical image-based examination. In 
this regard, image processing introduced a promising strategy to facilitate tumor grading and staging, while diminishing unnecessary expenses. Conventional image processing and machine learning techniques require extensive pre-processing, segmentation and manual extraction of specific visual features before classification. However, deep learning approaches have exceeded human performance in visual tasks by utilization of automated hierarchical feature extraction and classification by multi layers, which could be applied for cancer diagnosis using tumor tissue slides.

The first application of the image processing on analytical pathology for cancer detection was introduced by True et al. ([55]), and showed the implication of morphological features in diagnostic methods for malignant tumors. They used a series of morphological features including area fraction, shape, size and object counting to detect cell abnormalities. A large body of evidences has been published concerning cancer detection using various image processing and machine learning techniques ([18], [11], [29], [56], [61], [45]). Application of these methods is limited due to manual feature extraction of the features. Deep learning approach offers an automated, accurate and sensitive method to feature extraction from medical images.

In this regard, the Neighboring Ensemble Predictor (NEP) coupled with Constrained Convolutional Neural Network (SC$\mathrm{CNN}$ ) could lead to nucleus detection in colon cancer ([45]). Moreover, AggNet system which is a combination of CNN and additional crowd-sourcing layer, successfully detected mitosis in breast cancer images ([1]). In agreement with this, four deep learning network architectures including GoogLeNet, AlexNet, VGG16 deep network ([58]) and ConvNet with 3,4 , and 6 layers $([13])$ were recently applied to identify breast cancer. The best example of using automated CAD system is a study conducted by Esteva and colleague on skin cancer detection using Inception V3, which was done to classify malignancy status ([18]). In addition to these, studies such as ([8], [34], [2], [33]) also showed that deep learning techniques are continuously being applicable to image-based medical diagnosis and improve the performance compared to traditional machine learning techniques.

Despite improvements in images analysis and interpretation, numerous questions related to the reliability and sensitivity of appropriate pathological diagnosis systems particularly for breast cancer classification remained to be answered. In particular, there were no significant, comprehensive and promising solutions for discrimination of breast cancer sub-types.

This study presents deep learning Inception and ResNet architectures to discriminate microscopic cancerous imaging. We demonstrate a highly accurate automatic framework for cancer detection and classification of its sub-types. Our framework also employs additional techniques for data augmentation and advanced pre-processing.

\section{APPROACH}

In this study, we developed and introduced an accurate and reliable computer-based techniques empowered with deep learning approaches to classify cancer types and breast cancer
TABLE I

NUMBER OF IMAGES IN EACH CLASS

\begin{tabular}{|l|c|c|c|}
\hline \multicolumn{1}{|c|}{ Classes } & Sub-classes & Training & Testing \\
\hline $\begin{array}{l}\text { Cancers } \\
(6,402)\end{array}$ & $\begin{array}{c}\text { breast }(1,670) \text {, bladder }(1,870), \\
\text { lymphoma (1,560), lung (1,302) }\end{array}$ & 5,502 & 900 \\
\hline Breast cancer (7,909) & benign (2480), malignant (5,429) & 7,100 & 809 \\
\hline $\begin{array}{l}\text { Malignant } \\
(5,429)\end{array}$ & $\begin{array}{c}\text { ductal-carcinoma (3,451), lobular-carcinoma (626), } \\
\text { mucinous-carcinoma (792), papillary-carcinoma (560) }\end{array}$ & 4,879 & 550 \\
\hline $\begin{array}{l}\text { Malignant } \\
\text { Augmented }(9,394)\end{array}$ & $\begin{array}{c}\text { ductal-carcinoma (3,451), lobular-carcinoma (1,881), } \\
\text { mucinous-carcinoma (2,379), papillary-carcinoma (1,683) }\end{array}$ & 8,394 & 1,000 \\
\hline $\begin{array}{l}\text { Benign } \\
(2,480)\end{array}$ & $\begin{array}{c}\text { adenosis(444), fibroadenoma (1,014), } \\
\text { phyllodes-tumor (453), tubular-adenoma (569) }\end{array}$ & 2,220 & 260 \\
\hline $\begin{array}{l}\text { Benign } \\
\text { Augmented }(7,452)\end{array}$ & $\begin{array}{c}\text { adenosis (1,335), fibroadenoma (3,045), } \\
\text { phyllodes-tumor (1,362), tubular-adenoma (1,710) }\end{array}$ & 6,652 & 800 \\
\hline
\end{tabular}

sub-types from histopathological images derived from Hematoxylin and eosin stain (H\&E) and Immunohistochemistry (IHC) slides.

Our framework contains five steps: a) Image acquisition and conversion to JPEG/RGB channels. b) Data augmentation (section III-C. c) Deep learning pre-processing (section III-D). d) Transfer learning and fine-tuning pre-trained models (section III-E. e) Hierarchical feature extraction and classification with Inception and ResNet networks (sections III-F). All steps have been illustrated in figure 1 .

\section{Materials And Methods}

\section{A. Data-sets}

Data-sets were collected from two sources of cancer types and breast cancer sub-types including Tissue Micro Array (TMA) database ([23]) and BreaKHis (The Breast Cancer Histopathological Images) ([48]). 6,402 TMA histopathological images were applied across lung, breast, lymphoma, and bladder cancer tissues. BreaKHis 7,909 pathological breast cancer images (2,480 benign and 5,429 malignant images, each with different magnification of 40X, 100X, 200X, and 400X) from 82 patients were selected for sub-types classification. Our data-set contained four distinct histological sub-types of benign breast tumors: adenosis, fibroadenoma, phyllodes tumor, and tubular adenona; as well as four malignant tumors: ductal carcinoma, lobular carcinoma, mucinous carcinoma and papillary carcinoma (Table (1). It should be note that approximately $85 \%$ of available data were randomly chosen to construct the learning set. The remaining $15 \%$ of the data were used for performance evaluation.

\section{B. Color-map Selection}

In this work, we used RGB color-map to preserve tissue structures and different features of histophatological images.

\section{Data Augmentation}

Data augmentation is essential step to have enough diverse samples and learn a deep network from the images. Several studies investigated the role of data augmentation in deep learning ([44], [59], [16]). We considered data augmentation for breast cancer sub-types due to difference in the number of images among different sub-type classes. Technically, data augmentation was accomplished on data acquired from Augmentor Python library ([5] see supplementary material) and included random resizing, rotating, cropping, and flipping methods (Figure 2). 


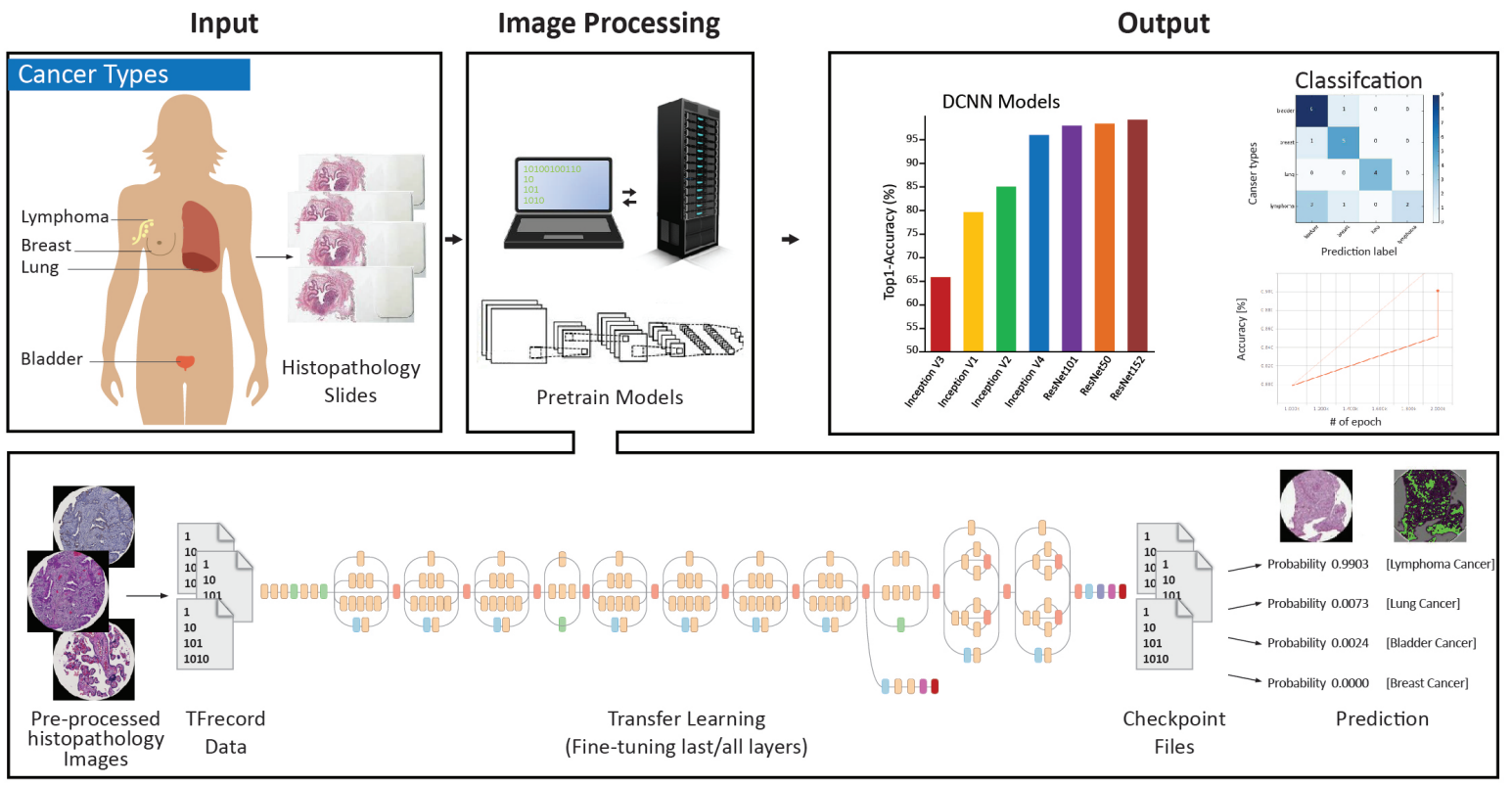

Fig. 1. Study work-flow; Data gathering, Image capturing, and Deep learning approaches using per-trained models. Data gathering included captured image from two individual TMA and BreakHis sources saved in JPEG format. Preprocessing is the next step followed by deep learning techniques to extract unique presentation for each separated cancer input.

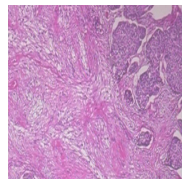

Image

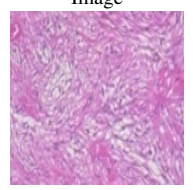

Crop
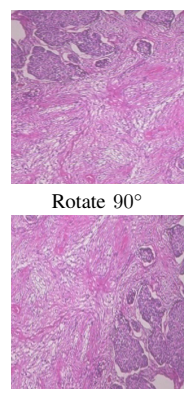

Flip
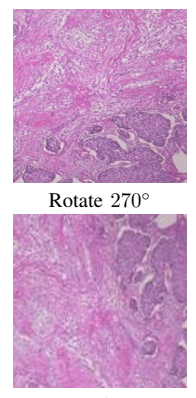

Resize
Fig. 2. Data augmentation techniques including rotating, cropping, flipping, resizing. Augmentor $([\overline{5]})$ rotated input image into 90 and 270 degrees. Then, image flipped top-bottom to right with 0.8 probability. Next image was cropped with probability of 1 and percentage area of 0.5 . Finally, input image was resized with width $=120$ and height $=120$

\section{Pre-Processing Steps}

Color map selection and data augmentation were followed by pre-processing steps as a preliminary recommended phase to prepare data for further feature extraction and analysis.

Previous studies proposed different pre-processing methods because of the nature of their data ([38], [10], [35], [32], [31]). This work proposed a series of calculation, divided into five steps. The first step focused on JPEG file decoder, followed by TFRecord ([21]) format conversion based on Protocol Buffers ([17], [22], [52]). In third step, TFRecords were normalized to $[0,1]$. Afterwards, whole image bounding box were re-sized to $299 \times 299 \times 3$ or $224 \times 224 \times 3$ according to the recommended model image size for Inception and ResNet architectures ([50], [49], [26]). Finally, as Inception and ResNet pre-processing, input training images were randomly flipped left to right horizontally and then cropped to create image summaries to display the different transformations on images. In order to improve power of learning and to make the network invariant to aspects of the image that do not affect the label, color distortion with permutation of four hue, brightness, saturation and contrast adjustment operations were applied. On the other hand, in the evaluation step, all images were normalized, cropped and re-sized to specific height and width (Figure 3).

\section{E. Transfer Learning}

Transfer learning is defined as exporting knowledge from previously learned source to a target task ([14], [62], [24], [3]). Learning from clinical images from scratch is often not the most practical strategy due to its computational cost, convergence problem ([51]), and insufficient number of highquality labeled samples. A growing body of experiments have investigated pre-trained models in the presence of limited learning samples ([63], [15], [43]).

Pre-trained ConvNets alongside fine-tuning and transfer learning lead to faster convergence and outperform training from scratch ([54], [51], [60]). Our target data-set (with 6402 cancer type and 7909 breast cancer sub-types histopathological images) is obviously smaller than the used reference data-set (ImageNet; training data with $1.2 \mathrm{M}$ ([53]).

Therefore, we initialized weight of different layers of our proposed network by using ImageNet Inception and ResNet pre-trained models. Then, we employed last layer fine-tuning on cancer images data set. Therefore, the ImageNet pre-trained weights were preserved while the last fully connected layer was updated continuously. Since, the cancer data-sets analyzed here are large and very different from ImageNet, the full layer fine-tuning was applied to compare accurately classification of cancers with the last layer fine tuning $([53])$ (Table S1) 


\section{F. Inception and ResNet Architectures}

Among various deep learning methods, we considered Inceptions and ResNet architectures. It is well understood that Inception models migrated from fully- to sparsely-connected architectures. In order to add more non-linearity capability, Inception module technically included $1 \times 1$ factorized convolutional neural networks followed by the rectified linear unit (ReLU). Also, a $3 \times 3$ convolutional layer was employed. Auxiliary logits with a combination of average pool, convolutional $1 \times 1$, fully connected, and softmax activation was applied to preserve the low-level detail features and tackle vanishing gradient problem in last layers. ResNet permanently utilized shortcut connections between shallow and deep networks to control and adjust training error rate ([49]).

This study examined different frameworks of Inception (V1, V2, V3, and V4) and ResNet (V1 50, V1 101, and V1 152) ([50], [49], [26]) on cancer digital images. Furthermore, RMSProp adaptive learning rate ([27], [42]) was applied with start- (0.001), decay- (0.9), and end-points (0.0001) settings. Because of insufficient number of available histopathological cancer images (section III-A) compared to numerous model parameters (up to 5 million in Inception and 10 million in ResNet), dropout regularization and batch normalization ([28]) were applied with batch sizes of 32 in training and 100 in evaluation steps.

\section{G. Computerized System Configuration}

Deep learning training with extreme number of network parameters, computational tasks and large data-sets was significantly accelerated by a single computing platform with following specifications: model: HP DL380 G9, CPU: 2x E52690v4 (35 MB L3 Cache, $2.6 \mathrm{GHz}, 14 \mathrm{C}$ ), RAM: 64 GB (8 $\times 8$ GB) RAM DDR4 $2133 \mathrm{MHz}$, HDD: 146 GB HDD 7.2k, GPU: ASUS GeForce GTX 1080, $1733 \mathrm{MHz}, 2560$ CUDA Cores, 8GB GDDR5 with CentOS 7.2 64-bit operating system and Python 3.5.3. In addition, The GPU-enabled version of TensorFlow required CUDA 8.0 Toolkit and cuDNN v5.1 ([36], [21]). All GPU necessary settings and details were obtained from TensorFlow and TFslim documentations and NVIDIA GPUs support ([21]).

\section{RESUlts}

The results were divided into following parts. a) Cancer types classification. b) Cancers were categorized as malignant and benign types. c) Malignant and benign samples were classified into their related four sub-types (sub-section [III-A].

Several standard performance terms such as true positive (TP), false positive (FP), true negative (TN), false negative (FN), accuracy (ACC), precision (P), AUC and sensitivity (S) were isolated from the confusion matrix ([46]).

\section{A. Classification of Cancer Types}

A $4 \times 4$ confusion matrix was used to represent prediction results of the set of four cancer pathological samples (subsection [III-A. The matrices were built on four rows and four columns: breast, lung, bladder, and lymphoma representing the
TABLE II

FINE-TUNING THE LAST LAYER FOR DIFFERENT MODELS IN CANCER TYPE CLASSIFICATION

\begin{tabular}{|l|c|c|c|c|c|c|c|c|c|}
\hline Model name & Epochs & ACC & TP & TN & FP & FN & P & AUC & S \\
\hline Inception V1 & $\mathbf{3 , 0 0 0}$ & $\mathbf{0 . 9 1 7}$ & 580 & 252 & 14 & 54 & $\mathbf{0 . 9 7 6}$ & $\mathbf{0 . 9 1 7}$ & 0.914 \\
\hline Inception V2 & 3,000 & 0.848 & 516 & 252 & 14 & 118 & 0.973 & 0.874 & 0.813 \\
\hline Inception V3 & 3,000 & 0.884 & 568 & 244 & 22 & 66 & 0.962 & 0.869 & 0.895 \\
\hline Inception V4 & 3,000 & 0.871 & 542 & 258 & 8 & 92 & 0.985 & 0.905 & 0.854 \\
\hline ResNet V1 50 & $\mathbf{3 , 0 0 0}$ & $\mathbf{0 . 9 9 3}$ & 630 & 266 & 0 & 4 & $\mathbf{1}$ & $\mathbf{0 . 9 9 6}$ & 0.993 \\
\hline ResNet V1 101 & $\mathbf{3 , 0 0 0}$ & $\mathbf{0 . 9 9 5}$ & 625 & 272 & 1 & 1 & $\mathbf{0 . 9 9 8}$ & $\mathbf{0 . 9 9 4}$ & 0.998 \\
\hline ResNet V1 152 & $\mathbf{3 , 0 0 0}$ & $\mathbf{0 . 9 9 2}$ & 623 & 272 & 1 & 4 & $\mathbf{0 . 9 9 8}$ & $\mathbf{0 . 9 9 3}$ & 0.993 \\
\hline
\end{tabular}

TABLE III

FINE-TUNING ALL LAYERS FOR DIFFERENT MODELS IN CANCER TYPE CLASSIFICATION

\begin{tabular}{|l|c|c|c|c|c|c|c|c|c|}
\hline Model name & Epochs & ACC & TP & TN & FP & FN & P & AUC & S \\
\hline Inception V1 & 2,000 & 0.793 & 531 & 254 & 1 & 114 & 0.998 & 0.91 & 0.823 \\
\hline Inception V1 & $\mathbf{3 , 0 0 0}$ & $\mathbf{0 . 9 7 1}$ & 624 & 258 & 8 & 10 & $\mathbf{0 . 9 8 7}$ & $\mathbf{0 . 9 8}$ & 0.984 \\
\hline Inception V2 & 2,000 & 0.86 & 621 & 172 & 100 & 7 & 0.861 & 0.753 & 0.988 \\
\hline Inception V2 & 3,000 & 0.935 & 630 & 232 & 34 & 4 & 0.948 & 0.972 & 0.993 \\
\hline Inception V3 & 2,000 & 0.65 & 628 & 17 & 255 & 0 & 0.7118 & 0.753 & 1 \\
\hline Inception V3 & 3,000 & 0.764 & 632 & 88 & 178 & 2 & 0.78 & 0.842 & 0.996 \\
\hline Inception V4 & 2,000 & 0.851 & 618 & 172 & 94 & 16 & 0.867 & 0.86 & 0.974 \\
\hline Inception V4 & 3,000 & 0.877 & 633 & 159 & 108 & 0 & 0.854 & 0.855 & 1 \\
\hline ResNet V1 50 & $\mathbf{2 , 0 0 0}$ & $\mathbf{0 . 9 8 8}$ & 627 & 263 & 10 & 0 & $\mathbf{0 . 9 8 4}$ & $\mathbf{0 . 9 8 1}$ & 1 \\
\hline ResNet V1 50 & $\mathbf{3 , 0 0 0}$ & $\mathbf{0 . 9 9 8}$ & 627 & 272 & 1 & 0 & $\mathbf{0 . 9 9 8}$ & $\mathbf{0 . 9 9 6}$ & 1 \\
\hline ResNet V1 101 & $\mathbf{2 , 0 0 0}$ & $\mathbf{0 . 9 8 3}$ & 616 & 273 & 0 & 11 & $\mathbf{1}$ & $\mathbf{0 . 9 9 1}$ & 0.982 \\
\hline ResNet V1 101 & $\mathbf{3 , 0 0 0}$ & $\mathbf{0 . 9 9 6}$ & 626 & 273 & 0 & 1 & $\mathbf{1}$ & $\mathbf{0 . 9 9 9}$ & 0.998 \\
\hline ResNet V1 152 & $\mathbf{2 , 0 0 0}$ & $\mathbf{0 . 9 9 2}$ & 623 & 272 & 1 & 4 & $\mathbf{0 . 9 9 8}$ & $\mathbf{0 . 9 9 3}$ & 0.993 \\
\hline ResNet V1 152 & $\mathbf{3 , 0 0 0}$ & $\mathbf{0 . 9 9 6}$ & 626 & 273 & 0 & 1 & $\mathbf{1}$ & $\mathbf{0 . 9 9 9}$ & 0.998 \\
\hline
\end{tabular}

known cancer classes. Statistical performance measurement of each cancer type and different deep learning frameworks (section [II-F) were summarized in Tables III and III] The result indicated that ResNet V1 50 and fine-tuning all layers classified $99.8 \%$ of known cancer types. While this rate decreased to $99.6 \%$ for ResNet V1 101/152 fine-tuning all layers with 3,000 epochs (Table III). ResNet V1 101 with 3,000 epochs and fine-tuning last layer had an accuracy rate of $99.5 \%$ (Table II). The ResNet models showed significantly increased accuracy for four cancer type classification compared to the all Inception structures, whereas Inception V1 with 3,000 epochs and fine-tuning all layer showed $97.1 \%$ accuracy at best. Additionally, there was an obvious difference in false positive values between Inception structures and ResNets. Furthermore, on average less false positive results were obtained by the ResNet (0.3) in comparison to the Inception models (82) with 3,000 epochs (Table III). The Cohens unweighted kappa coefficient statistic ([40]) of the Inception V1, V2, V3 and V4 fine-tuning all layers with 3,000 epochs were $0.94,0.94,0.82$ and 0.84 respectively,while those of the ResNet models were above 0.97 . The Inception networks were able to correctly identify four types of cancer with an accuracy ranging between $76.4 \%$ and $97.1 \%$, compared to the ResNet networks with ranging between $98.3 \%$ and $99.8 \%$ (Table III 3,000 epochs).

\section{B. Malignant and Benign Breast Cancer}

The breast cancer data (section [III-A and Table I) were categorized into malignant and benign groups. Using an $90 \%$ training set and 10\% test set,the ResNet V1 101 fine-tuning all layers, correctly classified malignant and benign cancer types with $98.4 \%$ confidence (Table IV). This performance for ResNet V1 50 with fine-tuning all layers and ResNet V1 101 with fine-tuning the last layer decreased to $97.8 \%$ and $94.1 \%$ respectively (Tables S2 and IV).

Inception V2 with fine-tuning all layers, indicated the maximum accuracy (94.1\%) among the Inception architectures 


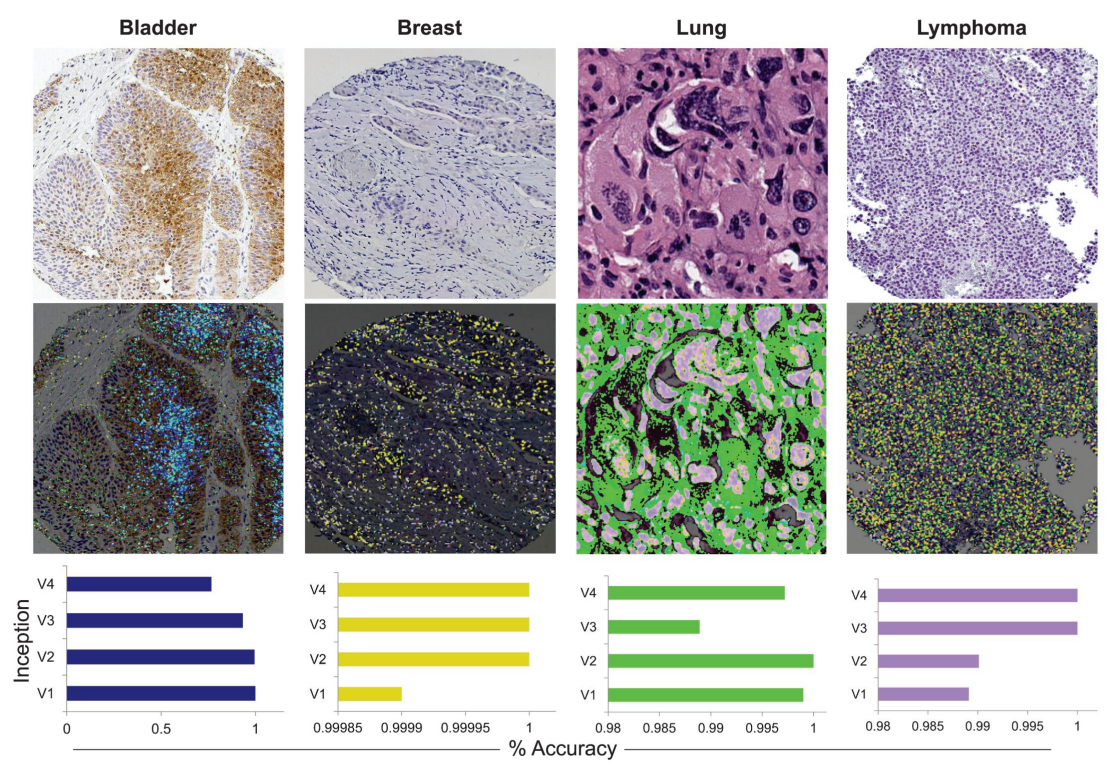

Fig. 3. From top to bottom: histopathological images from four cancer types were used as input data. Preprocessing techniques were applied to extract precise learned features. Accuracy of Inception V1 to V4 classification was presented as bar plots

TABLE IV

FINE-TUNING ALL LAYERS FOR DIFFERENT MODELS IN BREAST CANCER CLASSIFICATION

\begin{tabular}{|l|c|c|c|c|c|c|c|c|c|}
\hline Model name & Epochs & ACC & TP & TN & FP & FN & P & AUC & S \\
\hline Inception V1 & 3,000 & 0.936 & 553 & 290 & 9 & 48 & 0.983 & 0.945 & 0.92 \\
\hline Inception V2 & $\mathbf{3 , 0 0 0}$ & $\mathbf{0 . 9 4 1}$ & 593 & 254 & 45 & 8 & $\mathbf{0 . 9 2 9}$ & $\mathbf{0 . 9 1 8}$ & 0.986 \\
\hline Inception V3 & 3,000 & 0.822 & 500 & 294 & 5 & 101 & 0.99 & 0.907 & 0.831 \\
\hline Inception V4 & 3,000 & 0.777 & 403 & 297 & 2 & 198 & 0.995 & 0.831 & 0.67 \\
\hline ResNet V1 50 & $\mathbf{3 , 0 0 0}$ & $\mathbf{0 . 9 7 8}$ & 591 & 290 & 9 & 10 & $\mathbf{0 . 9 8 5}$ & $\mathbf{0 . 9 7 6}$ & 0.983 \\
\hline ResNet V1 101 & $\mathbf{3 , 0 0 0}$ & $\mathbf{0 . 9 8 4}$ & 594 & 292 & 7 & 7 & $\mathbf{0 . 9 8 8}$ & $\mathbf{0 . 9 8 2}$ & 0.988 \\
\hline ResNet V1 152 & $\mathbf{3 , 0 0 0}$ & $\mathbf{0 . 9 8 7}$ & 593 & 296 & 3 & 8 & $\mathbf{0 . 9 9 4}$ & $\mathbf{0 . 9 8 8}$ & 0.986 \\
\hline
\end{tabular}

(Table IV). Moreover, the results showed on average less false positive in the ResNet (6.3) compared to the Inception models (15.25) (Table IV).

\section{Breast Cancer Sub-types Classification}

In order to create a framework for approval classification capability, we considered wide varieties of similar and complex histopathological images related to different sub-types of breast cancer. Since the benign and malignant groups were well separated from each other (section IV-B), further, we assessed pre-trained Inception and ResNet models to classify benign and malignant related sub-types. According to our results, the accuracy of analysis for benign sub-types resulted to classification of adenosis, fibroadenoma, phyllodes-tumor, and tubular-adenoma (Tables S3 and V].

In case of malignant classification, accuracy analysis for associated sub-types (on test sets) resulted in $96.4 \%$, and $94.6 \%$ for ResNet V1 152 and ResNet V1 50 with fine-tuning all layers respectively (Table VIII. Moreover, an accuracy rate of $90 \%$ for ResNet V1 152 with fine-tuning the last layer is also acceptable (Table VI).
As represented in (Table VII), the ResNet networks output illustrated significantly higher level of accuracy than other Inception structures in which Inception V1 with 3, 000 epochs and fine-tuning all layers showed $86.6 \%$ marked as less accurate method in terms of malignant cancer sub-types classification. Additionally, there has been an obvious difference in false positive values between Inception structures and ResNets with average of 0.75 and 0.33 , respectively (Table VII].

A $4 \times 4$ confusion matrix was used to represent different possibilities of the set of instances. The matrices represented distribution of the ductal-carcinoma, lobular-carcinoma, mucinous-carcinoma, papillary-carcinoma in different classes. It was well evidenced that increases in the number of epochs could improve the accuracy of classification. Our findings (Tables V] and VII suggest that ResNet with higher epochs was highly accurate (for example; $96.5 \%$ and $98.5 \%$ ResNet 101 with 6,000 epochs) for classification of specific sub-types of breast cancer (Figure 4 .

TABLE V

FINE-TUNING ALL LAYERS FOR DIFFERENT MODELS IN AUGMENTED BENIGN DATA.

\begin{tabular}{|l|c|c|c|c|c|c|c|c|c|}
\hline Model name & Epochs & ACC & TP & TN & FP & FN & P & AUC & S \\
\hline Inception V1 & 3,000 & 0.696 & 660 & 88 & 48 & 4 & 0.932 & 0.779 & 0.993 \\
\hline Inception V2 & $\mathbf{3 , 0 0 0}$ & $\mathbf{0 . 7 2 3}$ & 660 & 95 & 41 & 4 & $\mathbf{0 . 9 4 1}$ & $\mathbf{0 . 8 7 5}$ & 0.993 \\
\hline Inception V3 & 3,000 & 0.512 & 638 & 44 & 92 & 26 & 0.873 & 0.629 & 0.96 \\
\hline Inception V4 & 3,000 & 0.54 & 651 & 53 & 83 & 13 & 0.886 & 0.404 & 0.98 \\
\hline ResNet V1 50 & $\mathbf{3 , 0 0 0}$ & $\mathbf{0 . 9 4 8}$ & 661 & 132 & 4 & 3 & $\mathbf{0 . 9 9 3}$ & $\mathbf{0 . 9 7 3}$ & 0.995 \\
\hline ResNet V1 101 & $\mathbf{3 , 0 0 0}$ & $\mathbf{0 . 9 3 3}$ & 658 & 132 & 4 & 6 & $\mathbf{0 . 9 9 3}$ & $\mathbf{0 . 9 7 1}$ & 0.99 \\
\hline ResNet V1 152 & $\mathbf{3 , 0 0 0}$ & $\mathbf{0 . 9 4 5}$ & 662 & 129 & 7 & 2 & $\mathbf{0 . 9 8 9}$ & $\mathbf{0 . 9 8}$ & 0.996 \\
\hline ResNet V1 101 & $\mathbf{6 , 0 0 0}$ & $\mathbf{0 . 9 6 5}$ & 659 & 134 & 2 & 5 & $\mathbf{0 . 9 9 6}$ & $\mathbf{0 . 9 9 2}$ & 0.992 \\
\hline
\end{tabular}



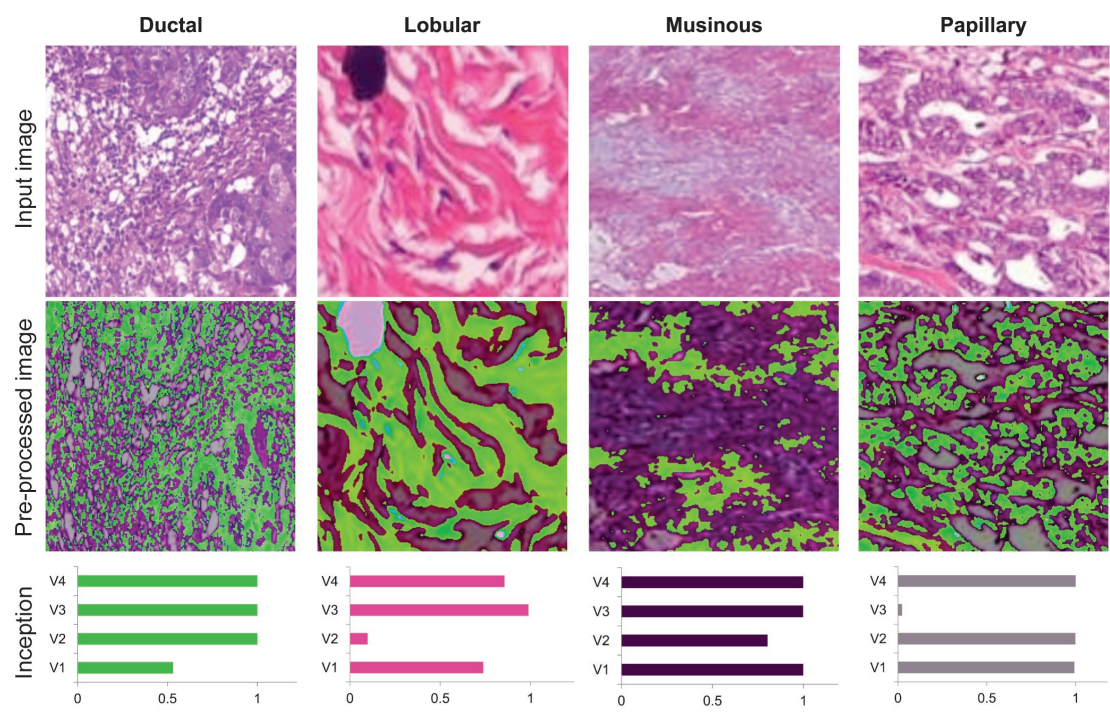

v3 I

vis

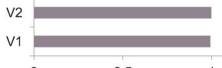

Fig. 4. From top to bottom: histopathological images from four cancer types were used as input data. Preprocessing techniques were applied to extract precise learned features. Accuracy of Inception V1 to V4 classification was presented as bar plots

TABLE VI

FINE-TUNING THE LAST LAYER FOR DIFFERENT MODELS IN AUGMENTED MALIGNANT DATA

\begin{tabular}{|l|c|c|c|c|c|c|c|c|c|}
\hline Model name & Epochs & ACC & TP & TN & FP & FN & P & AUC & S \\
\hline Inception V1 & $\mathbf{3 , 0 0 0}$ & $\mathbf{0 . 7 9 4}$ & 631 & 366 & 1 & 2 & $\mathbf{0 . 9 9 8}$ & $\mathbf{0 . 9 9 7}$ & 0.996 \\
\hline Inception V2 & 3,000 & 0.647 & 456 & 366 & 1 & 177 & 0.997 & 0.857 & 0.72 \\
\hline Inception V3 & 3,000 & 0.74 & 632 & 365 & 2 & 1 & 0.996 & 0.995 & 0.998 \\
\hline Inception V4 & 3,000 & 0.745 & 624 & 362 & 5 & 9 & 0.992 & 0.981 & 0.985 \\
\hline ResNet V1 50 & $\mathbf{3 , 0 0 0}$ & $\mathbf{0 . 8 9 3}$ & 632 & 367 & 0 & 18 & $\mathbf{1}$ & $\mathbf{0 . 9 9 9}$ & 0.998 \\
\hline ResNet V1 101 & $\mathbf{3 , 0 0 0}$ & $\mathbf{0 . 8 7 2}$ & 630 & 367 & 0 & 3 & $\mathbf{1}$ & $\mathbf{0 . 9 9 7}$ & 0.995 \\
\hline ResNet V1 152 & $\mathbf{3 , 0 0 0}$ & $\mathbf{0 . 9}$ & 627 & 367 & 1 & 5 & $\mathbf{0 . 9 9 8}$ & $\mathbf{0 . 9 9 5}$ & 0.992 \\
\hline
\end{tabular}

TABLE VII

FINE-TUNING ALL LAYERS FOR DIFFERENT MODELS IN AUGMENTED MALIGNANT DATA

\begin{tabular}{|l|l|c|c|c|c|c|c|c|c|}
\hline Model name & Epochs & ACC & TP & TN & FP & FN & P & AUC & S \\
\hline Inception V1 & $\mathbf{3 , 0 0 0}$ & $\mathbf{0 . 8 6 6}$ & 631 & 368 & 0 & 1 & $\mathbf{1}$ & $\mathbf{0 . 9 9 9}$ & 0.998 \\
\hline Inception V2 & 3,000 & 0.565 & 283 & 366 & 1 & 350 & 0.996 & 0.721 & 0.447 \\
\hline Inception V3 & 3,000 & 0.739 & 633 & 367 & 0 & 0 & 1 & 1 & 1 \\
\hline Inception V4 & 3,000 & 0.799 & 634 & 364 & 2 & 0 & 0.996 & 0.999 & 1 \\
\hline ResNet V1 50 & $\mathbf{3 , 0 0 0}$ & $\mathbf{0 . 9 4 6}$ & 624 & 367 & 1 & 8 & $\mathbf{0 . 9 9 8}$ & $\mathbf{0 . 9 9 3}$ & 0.987 \\
\hline ResNet V1 101 & $\mathbf{3 , 0 0 0}$ & $\mathbf{0 . 8 4 4}$ & 529 & 367 & 0 & 104 & $\mathbf{1}$ & $\mathbf{0 . 9 1 7}$ & 0.835 \\
\hline ResNet V1 152 & $\mathbf{3 , 0 0 0}$ & $\mathbf{0 . 9 6 4}$ & 629 & 367 & 0 & 4 & $\mathbf{1}$ & $\mathbf{0 . 9 9 6}$ & 0.993 \\
\hline ResNet V1 101 & $\mathbf{6 , 0 0 0}$ & $\mathbf{0 . 9 8 5}$ & 633 & 367 & 0 & 0 & $\mathbf{1}$ & $\mathbf{1}$ & 1 \\
\hline
\end{tabular}

\section{Discussion}

Breast cancer multi-classification aim to identify sub-classes of breast cancer (adenosis, fibroadenoma, phyllodes tumor, tubular adenoma, ductal carcinoma, lobular carcinoma, mucinous carcinoma, and papillary carcinoma) among samples found in broad variability of resolution image appearances, high coherency of cancerous cells, and extensive inhomogeneity of color distribution. This work examined data gathered from TMA and BreaKHis data-sets (see section III) to classify cancer types and breast sub-types. Previous studies ([30], [47], [48]) focused on binary benign-malignant classification and did not perform further quantitative assessment. In this work, we introduced automated breast cancer multi-classification methods. We suggested a generic CAD framework based on deep networks for learning histopathology images to avoid hand-crafted pathological features. In this study, we compared the performance of Inception and ResNet deep learning models using transfer learning strategy on several large image datasets. We found that deep ResNet models were more sensitive and reliable than Inception in all tested cancer data-sets. We combined different magnification including 40X, 100X, 200X and 400X to generate comprehensive, independent and scalable system while a large number of previous studies employed single magnification level ([7], [30]). Several other studies ([7], [25], [48], [4]) also investigated multiple magnifications of medical images. However, these approaches examined different classifiers for each magnification level and also had medical laboratory limitations to capture required multiple magnification to gather image training samples.

In recent comparative studies, ([48], [30], [7]), conventional machine learning (SVM, KNN, QDA, ASSVM, SSVMSCAD, etc) along with hand-crafted feature extraction were used. The results were evaluated at various magnifications (i.e. 40X, 100X, 200X and 400X). To data, the benign and malignant classification had significantly high accuracy rates ranging from 90 to $93 \%$. In addition, to classify benign and malignant, AlexNet deep learning approach with numerous learning parameters resulted in an accuracy rate of $90 \%$ (447). Moreover, Han and colleagues (Han et al., 2017), reported a deep learning-based multi classification of breast cancer with an average accuracy rate of the $93.2 \%$.

In conclusion, the ResNet frameworks with $99.8 \%, 98.7 \%, 94.8 \%$, and $96.4 \%$ accuracy for four cancer types, two main breast cancer types, benign and malignant related sub-types and trivial false positive average values ( 0.3 out of 900 for four cancer types, 6.3 out of 809 for all breast cancer, 5 out of 800 for benign and 0.3 out of 1000 for malignant) were able to examine histopathological images obtained by different imaging devices with different magnification levels. This multi-classification system relieves the pathologists and medical experts workloads regarding 
to analyze and interpretation of the histopathological slides for assisting the doctors to choose more efficient therapeutic approaches.

\section{CONCLUSion AND Future Work}

Using deep learning ResNet approach with specific settings for cancer detection is an effective and reliable strategy compared to the conventional approaches. This work concentrated on the application of the proposed frameworks to cancer subtypes detection. In this research, various Inception and ResNet deep learning classifications are presented and the use of these theories is outlined. The findings are expected to be more comprehensively evaluated and discussed by future works considering different deep learning semantic segmentation algorithms (i.e., U-net: convolutional networks for biomedical image segmentation ([41]) and DeepLab v3 ([9])). The empirical findings of this study provided a better understanding of deep learning in medical applications. The framework principals could extend in the field of pathological analysis and computer-assisted diagnosis using medical images.

\section{ACKNOWLEDGMENT}

We would like to acknowledge Dr. Mahdi Pourfath from the University of Tehran and his team for a wide range of resources on GPU programming. We would like to appreciate comments and ideas by Dr. Ali Sharifi Zarchi.

\section{FUNDING}

This work has been supported in part by the Iranian National Elite Foundation and grants provided by Royan Institute to Mehdi Totonchi and a start-up fund from Weill Cornell Medicine to Iman Hajirasouliha.

\section{REFERENCES}

[1] S. Albarqouni, C. Baur, F. Achilles, V. Belagiannis, S. Demirci, and N. Navab. Aggnet: Deep learning from crowds for mitosis detection in breast cancer histology images. IEEE Transactions on Medical Imaging, 35(5):1313-1321, May 2016.

[2] M. Aubreville, C. Knipfer, N. Oetter, C. Jaremenko, E. Rodner, J. Denzler, C. Bohr, H. Neumann, F. Stelzle, and A. Maier. Automatic classification of cancerous tissue in laserendomicroscopy images of the oral cavity using deep learning. arXiv preprint arXiv:1703.01622, 2017.

[3] M. T. Bahadori, Y. Liu, and D. Zhang. A general framework for scalable transductive transfer learning. Knowledge and information systems, 38(1):61-83, 2014.

[4] N. Bayramoglu, J. Kannala, and J. Heikkilä. Deep learning for magnification independent breast cancer histopathology image classification. In Pattern Recognition (ICPR), 2016 23rd International Conference on, pages 2440-2445. IEEE, 2016.

[5] M. D. Bloic. Augmentor-image augmentation library in python for machine learning. https://github.com/mdbloice/Augmentor 2017. [The MIT License (MIT), Online; accessed 16-July-2017].

[6] L. Carey, E. Winer, G. Viale, D. Cameron, and L. Gianni. Triple-negative breast cancer: disease entity or title of convenience? Nature reviews Clinical oncology, 7(12):683-692, 2010.

[7] A. Chan and J. A. Tuszynski. Automatic prediction of tumour malignancy in breast cancer with fractal dimension. Open Science, 3(12):160558, 2016.

[8] H. Chang. Skin cancer reorganization and classification with deep neural network. arXiv preprint arXiv:1703.00534, 2017.

[9] L.-C. Chen, G. Papandreou, F. Schroff, and H. Adam. Rethinking atrous convolution for semantic image segmentation. arXiv preprint arXiv:1706.05587, 2017.
[10] P. F. Christ, M. E. A. Elshaer, F. Ettlinger, S. Tatavarty, M. Bickel, P. Bilic, M. Rempfler, M. Armbruster, F. Hofmann, M. D́Anastasi, et al. Automatic liver and lesion segmentation in CT using cascaded fully convolutional neural networks and 3D conditional random fields. In International Conference on Medical Image Computing and ComputerAssisted Intervention, pages 415-423. Springer, 2016.

[11] D. C. Cireşan, A. Giusti, L. M. Gambardella, and J. Schmidhuber. Mitosis Detection in Breast Cancer Histology Images with Deep Neural Networks, pages 411-418. Springer Berlin Heidelberg, Berlin, Heidelberg, 2013.

[12] M. Colleoni, N. Rotmensz, P. Maisonneuve, M. Mastropasqua, A. Luini, P. Veronesi, M. Intra, E. Montagna, G. Cancello, A. Cardillo, et al. Outcome of special types of luminal breast cancer. Annals of oncology, 23(6):1428-1436, 2011.

[13] A. Cruz-Roa, H. Gilmore, A. Basavanhally, M. Feldman, S. Ganesan, N. N. Shih, J. Tomaszewski, F. A. González, and A. Madabhushi. Accurate and reproducible invasive breast cancer detection in wholeslide images: A deep learning approach for quantifying tumor extent. Scientific Reports, 7:46450, 2017.

[14] H. Daume III and D. Marcu. Domain adaptation for statistical classifiers. Journal of Artificial Intelligence Research, 26:101-126, 2006.

[15] J. Donahue, Y. Jia, O. Vinyals, J. Hoffman, N. Zhang, E. Tzeng, and T. Darrell. Decaf: A deep convolutional activation feature for generic visual recognition. In International conference on machine learning, pages $647-655,2014$

[16] A. Dosovitskiy, J. T. Springenberg, and T. Brox. Unsupervised feature learning by augmenting single images. CoRR, abs/1312.5242, 2013.

[17] D. Eddelbuettel, M. Stokely, and J. Ooms. Rprotobuf: Efficient crosslanguage data serialization in r. arXiv preprint:1401.7372, 2014.

[18] A. Esteva, B. Kuprel, R. A. Novoa, J. Ko, S. M. Swetter, H. M. Blau, and S. Thrun. Dermatologist-level classification of skin cancer with deep neural networks. Nature, 542(7639):115-118, 2017.

[19] C. Fitzmaurice, C. Allen, R. M. Barber, L. Barregard, Z. A. Bhutta, H. Brenner, D. J. Dicker, O. Chimed-Orchir, R. Dandona, L. Dandona, et al. Global, regional, and national cancer incidence, mortality, years of life lost, years lived with disability, and disability-adjusted life-years for 32 cancer groups, 1990 to 2015: a systematic analysis for the global burden of disease study. JAMA oncology, 3(4):524-548, 2017.

[20] A. . Goldhirsch, W. Wood, A. Coates, R. Gelber, B. Thürlimann, H.-J. Senn, and P. members. Strategies for subtypesdealing with the diversity of breast cancer: highlights of the st gallen international expert consensus on the primary therapy of early breast cancer 2011. Annals of oncology, 22(8):1736-1747, 2011

[21] T. Google-Developers. Installing tensorflow on ubuntu. https://www. tensorflow.org/install/install_linux 2017.

[22] T. Google-Developers. Protocol buffers. https://developers.google.com/ protocol-buffers/?hl=en 2017.

[23] T. Google-Developers. Stanford tissue microarray database. https://tma. im/cgi-bin/home.pl 2017.

[24] L. Gui, R. Xu, O. Lu, J. Du, and Y. Zhou. Negative transfer detection in transductive transfer learning. International Journal of Machine Learning and Cybernetics, pages 1-13, 2017.

[25] Z. Han, B. Wei, Y. Zheng, Y. Yin, K. Li, and S. Li. Breast cancer multi-classification from histopathological images with structured deep learning model. Scientific Reports, 7, 2017.

[26] K. He, X. Zhang, S. Ren, and J. Sun. Deep residual learning for image recognition. In Proceedings of the IEEE conference on computer vision and pattern recognition, pages 770-778, 2016.

[27] G. Hinton, N. Srivastava, and K. Swersky. Rmsprop: Divide the gradient by a running average of its recent magnitude. Neural networks for machine learning, Coursera lecture 6e, 2012.

[28] S. Ioffe and C. Szegedy. Batch normalization: Accelerating deep network training by reducing internal covariate shift. In International Conference on Machine Learning, pages 448-456, 2015.

[29] A. Jalalian, S. B. Mashohor, H. R. Mahmud, M. I. B. Saripan, A. R. B. Ramli, and B. Karasfi. Computer-aided detection/diagnosis of breast cancer in mammography and ultrasound: a review. Clinical Imaging, 37(3):420 - 426, 2013.

[30] M. A. Kahya, W. Al-Hayani, and Z. Y. Algamal. Classification of breast cancer histopathology images based on adaptive sparse support vector machine. Journal of Applied Mathematics and Bioinformatics, 7(1):49, 2017.

[31] S. Kalouche. Vision-based classification of skin cancer using deep learning, 2015. conducted on Stanfords Machine Learning course (CS 229) taught by Professor Andrew Ng and John Duchi.

[32] K. Kamnitsas, C. Ledig, V. F. J. Newcombe, J. P. Simpson, A. D. Kane, D. K. Menon, D. Rueckert, and B. Glocker. Efficient multi-scale 3D 
CNN with fully connected CRF for accurate brain lesion segmentation. CoRR, abs/1603.05959, 2016.

[33] P. Khosravi, E. Kazemi, M. Imielinski, O. Elemento, and I. Hajirasouliha. Deep convolutional neural networks enable discrimination of heterogeneous digital pathology images. EBioMedicine.

[34] Y. Liu, K. Gadepalli, M. Norouzi, G. E. Dahl, T. Kohlberger, A. Boyko, S. Venugopalan, A. Timofeev, P. Q. Nelson, G. S. Corrado, et al. Detecting cancer metastases on gigapixel pathology images. arXiv preprint arXiv:1703.02442, 2017.

[35] D. Nie, H. Zhang, E. Adeli, L. Liu, and D. Shen. 3D deep learning for multi-modal imaging-guided survival time prediction of brain tumor patients. In International Conference on Medical Image Computing and Computer-Assisted Intervention, pages 212-220. Springer, 2016.

[36] T. NVIDIA-Developers. NVIDIA GPUs - The Engine of Deep Learning. https://developer.nvidia.com/deep-learning 2017.

[37] W. H. Organization et al. Tumours of the breast and female genital organs. World Health Organization Classification of Tumours: Pathology and Genetics of Tumours of the Breast and Female Genital Organs. Lyon: International Agency for Research in Cancer, 2003.

[38] S. Parisot, S. I. Ktena, E. Ferrante, M. Lee, R. G. Moreno, B. Glocker, and D. Rueckert. Spectral graph convolutions on population graphs for disease prediction. arXiv preprint:1703.03020, 2017.

[39] B. C. Pestalozzi, D. Zahrieh, E. Mallon, B. A. Gusterson, K. N. Price, R. D. Gelber, S. B. Holmberg, J. Lindtner, R. Snyder, B. Thurlimann, et al. Distinct clinical and prognostic features of infiltrating lobular carcinoma of the breast: combined results of 15 international breast cancer study group clinical trials. Journal of clinical oncology, 26(18):30063014, 2008.

[40] R. G. Pontius Jr and M. Millones. Death to kappa: birth of quantity disagreement and allocation disagreement for accuracy assessment. International Journal of Remote Sensing, 32(15):4407-4429, 2011.

[41] O. Ronneberger, P. Fischer, and T. Brox. U-net: Convolutional networks for biomedical image segmentation. In International Conference on Medical Image Computing and Computer-Assisted Intervention, pages 234-241. Springer, 2015.

[42] T. Schaul, S. Zhang, and Y. LeCun. No more pesky learning rates. In International Conference on Machine Learning, pages 343-351, 2013.

[43] P. Sermanet, D. Eigen, X. Zhang, M. Mathieu, R. Fergus, and Y. LeCun. Overfeat: Integrated recognition, localization and detection using convolutional networks. arXiv preprint arXiv:1312.6229, 2013.

[44] A. A. A. Setio, F. Ciompi, G. Litjens, P. Gerke, C. Jacobs, S. J. van Riel, M. M. W. Wille, M. Naqibullah, C. I. Sánchez, and B. van Ginneken. Pulmonary nodule detection in CT images: false positive reduction using multi-view convolutional networks. IEEE transactions on medical imaging, 35(5):1160-1169, 2016.

[45] K. Sirinukunwattana, S. E. A. Raza, Y. W. Tsang, D. R. J. Snead, I. A. Cree, and N. M. Rajpoot. Locality sensitive deep learning for detection and classification of nuclei in routine colon cancer histology images. IEEE Transactions on Medical Imaging, 35(5):1196-1206, May 2016.

[46] M. Sokolova and G. Lapalme. A systematic analysis of performance measures for classification tasks. Inf. Process. Manage., 45(4):427-437, July 2009.

[47] F. A. Spanhol, L. S. Oliveira, C. Petitjean, and L. Heutte. Breast cancer histopathological image classification using convolutional neural networks. In Neural Networks (IJCNN), 2016 International Joint Conference on, pages 2560-2567. IEEE, 2016.

[48] F. A. Spanhol, L. S. Oliveira, C. Petitjean, and L. Heutte. A dataset for breast cancer histopathological image classification. IEEE Transactions on Biomedical Engineering, 63(7):1455-1462, 2016.

[49] C. Szegedy, W. Liu, Y. Jia, P. Sermanet, S. E. Reed, D. Anguelov, D. Erhan, V. Vanhoucke, and A. Rabinovich. Going deeper with convolutions. CoRR, abs/1409.4842, 2014.

[50] C. Szegedy, V. Vanhoucke, S. Ioffe, J. Shlens, and Z. Wojna. Rethinking the inception architecture for computer vision. In Proceedings of the IEEE Conference on Computer Vision and Pattern Recognition, pages 2818-2826, 2016.

[51] N. Tajbakhsh, J. Y. Shin, S. R. Gurudu, R. T. Hurst, C. B. Kendall, M. B. Gotway, and J. Liang. Convolutional neural networks for medical image analysis: Full training or fine tuning? IEEE transactions on medical imaging, 35(5):1299-1312, 2016.

[52] TensorFlow-Group. A tool developer's guide to tensorflow model files. https://www.tensorflow.org/extend/tool_developers/. 2017.

[53] T. TensorFlow-Group. Tensorflow-slim image classification library. https://github.com/tensorflow/models/tree/master/slim 2016. [ Apache License, Version 2.0, Online; accessed 17-July-2017].

[54] S. Thrun and L. Pratt, editors. Learning to Learn. Kluwer Academic Publishers, Norwell, MA, USA, 1998.
[55] L. D. True. Morphometric applications in anatomic pathology. Human Pathology, 27(5):450 - 467, 1996.

[56] P. D. Velusamy and P. Karandharaj. Medical image processing schemes for cancer detection: A survey. In International Conference on Green Computing Communication and Electrical Engineering (ICGCCEE), pages 1-6, March 2014.

[57] G. Viale, N. Rotmensz, P. Maisonneuve, E. Orvieto, E. Maiorano, V. Galimberti, A. Luini, M. Colleoni, A. Goldhirsch, and A. S. Coates. Lack of prognostic significance of classic lobular breast carcinoma: a matched, single institution series. Breast cancer research and treatment, 117(1):211, 2009.

[58] D. Wang, A. Khosla, R. Gargeya, H. Irshad, and A. H. Beck. Deep learning for identifying metastatic breast cancer. arXiv preprint arXiv:1606.05718, 2016.

[59] R. Wu, S. Yan, Y. Shan, Q. Dang, and G. Sun. Deep image: Scaling up image recognition. arXiv preprint:1501.02876, 7(8), 2015.

[60] J. Yosinski, J. Clune, Y. Bengio, and H. Lipson. How transferable are features in deep neural networks? In Advances in neural information processing systems, pages 3320-3328, 2014.

[61] K.-H. Yu, C. Zhang, G. J. Berry, R. B. Altman, C. Ré, D. L. Rubin, and M. Snyder. Predicting non-small cell lung cancer prognosis by fully automated microscopic pathology image features. Nature communications, 7, 2016.

[62] B. Zadrozny. Learning and evaluating classifiers under sample selection bias. In Proceedings of the twenty-first international conference on Machine learning, page 114. ACM, 2004.

[63] M. D. Zeiler and R. Fergus. Visualizing and understanding convolutional networks. In European conference on computer vision, pages 818-833. Springer, 2014. 